\title{
Holographic constraints on generalized Rivlin-Ericksen fluid
}

\author{
Suvankar Dutta, ${ }^{*}$ Taniya Mandal, ${ }^{\dagger}$ and Sanhita Parihar \\ Indian Institute of Science Education and Research Bhopal, Bhopal Bypass, Bhopal 462066, India
}

(Received 26 November 2020; accepted 5 January 2021; published 21 January 2021)

\begin{abstract}
The Rivlin-Ericksen model is one of the oldest models in fluid dynamics to describe non-Newtonian properties. The model comes with two independent transports at second order. In this paper, we study the relativistic origin of the Rivlin-Ericksen fluid. Starting from a relativistic Weyl invariant uncharged fluid in $3+1$ dimensions, we reduce it over light-cone directions and obtain a generic nonrelativistic uncharged fluid in one lower dimension with all possible second-order terms in the constitutive relations. We observe that the Rivlin-Ericksen fluid is a subclass of our generalized nonrelativistic system. We also compute the holographic values of all the nonrelativistic second-order transports and find that three of them satisfy a universal constraint relation.
\end{abstract}

DOI: 10.1103/PhysRevD.103.026014

\section{INTRODUCTION}

Fluid dynamics, one of the oldest and widely studied subjects, is offering new surprises even today. A few experimental evidences and theoretical results in the recent past have created a lot of interest in the field of relativistic hydrodynamics and opened up new avenues for further investigation with many fundamental questions. One of the interesting results in this direction was the discovery of an anomalous transport coefficient in the context of the AdS/CFT correspondence [1,2]. Later in Ref. [3], it was shown that such a term in the constitutive relation (energymomentum tensor and other charge currents) is not only allowed by the symmetry but is also indispensable to preserve the second law of thermodynamics. This was the beginning of a new era in the field of relativistic hydrodynamics.

Nonrelativistic systems, on the other hand, are also interesting mainly because they are expected to be realized in low-energy experiments and condensed matter physics. Being an effective description of a nearly equilibrium interacting system, the constitutive relations of a fluid dynamic system (relativistic or nonrelativistic) are perturbatively written in derivative expansion of fluid variables. ${ }^{1}$

\footnotetext{
*suvankar@iiserb.ac.in

tmandal@iiserb.ac.in

*sanhita18@iiserb.ac.in

${ }^{1}$ In the relativistic system, consideration of second-order terms in conserved currents is essential because the first-order formalism is inconsistent with causality [4-6]. See Ref. [7] for a comparing discussion.

Published by the American Physical Society under the terms of the Creative Commons Attribution 4.0 International license. Further distribution of this work must maintain attribution to the author(s) and the published article's title, journal citation, and DOI. Funded by SCOAP ${ }^{3}$.
}

To write the independent derivative terms at every order in perturbative expansion, one has to follow a guiding principle. Such a principle could either be empirical or fundamental. For example, to a certain order in derivative expansion, one can include all the terms allowed by the symmetry of the system. In this paper, we follow a fundamental principle to write down the allowed independent terms systematically in the constitutive relations of a nonrelativistic uncharged fluid up to second order in derivative expansion. It is believed that all the interacting systems are relativistic at the microscopic level. A nonrelativistic system is an effective low-energy (velocity) description of an underlying relativistic system. Therefore, we start with a generic second-order relativistic fluid with all possible independent terms in energymomentum tensors allowed by the symmetries [8]. We then take the nonrelativistic limit and obtain a reduced nonrelativistic system with independent second derivative terms in its stress tensors and other conserved currents. Taking the nonrelativistic limit is not unique, and hence the nonrelativistic constitutive relations, in general, depend on how the limit is taken [9-17]. In this paper, we consider light-cone reduction [18-20] to obtain the nonrelativistic system.

Light-cone reduction reduces a relativistic field theory to a nonrelativistic field theory in one lower space dimension. This is because the symmetry algebra of the relativistic theory reduces to corresponding nonrelativistic symmetry algebra in one lower space dimension upon light-cone reduction. For example, the Poincaré algebra reduces to Galilean algebra, and conformal algebra boils down to Schrödinger algebra [21-25]. Therefore, lightcone reduction of relativistic constitutive equations renders the nonrelativistic constitutive equations for a fluid in one lower dimension. Light-cone reduction of relativistic 
fluids ${ }^{2}$ up to first order in derivative expansion has been discussed in Refs. [18-20]. Construction of a nonrelativistic entropy current was also discussed in Ref. [20]. A holographic computation of the nonrelativistic stress tensor and transports have been reported in Refs. [30,31]. However, all the works so far mainly considered constitutive relations up to first order in derivative expansions. In this paper, we consider a parity-odd uncharged relativistic fluid obeying Poincaré and Weyl symmetry in 3+1-dimensional flat spacetime and use the lightcone reduction to obtain a nonrelativistic fluid with Schrödinger symmetry in 2+1-dimensional flat space with generic second-order terms in constitutive relations.

The appearance of second and higher derivative terms in the constitutive relations of a nonrelativistic fluid has been well known for a long time. Such fluids are broadly known as non-Newtonian fluid. The Newtonian fluid possesses a linear relation between the viscous stress and rate of strain tensor. Explaining the properties of a more general class of fluid like honey, thick oils, paints, etc., requires relaxing the linear relation between stress and strain tensor, thus leading to the study of non-Newtonian fluid. One way to include such higher derivative terms in the stress tensor is by modifying the constitutive relations as suggested by experiments. The other approach is more fundamental-mainly based on symmetries. This was first studied by Reiner [32] in 1945. Later in 1948, Rivlin generalized Reiner's idea for isotropic fluid assuming that the stress tensor depends only on the velocity gradients and is invariant under a coordinate transformation [33]. This model, known as the Reiner-Rivlin model, explains non-Newtonian properties like the normal stress effect, centripetal pump effect, and Merrington effect. The model also predicts that in a simple shear flow the normal stresses in and perpendicular to the plane of shear are equal, which was in contradiction to observations. To explain the difference between two normal stresses, the form of the stress tensor requires being generalized and was given by Rivlin and Ericksen [34] in 1955. They assumed that the higher derivative terms in the stress tensor of an isotropic and homogeneous fluid depend on the gradient of velocity, acceleration, second acceleration, and so on and are also frame invariant. However, this model fails to explain gradual stress relaxation. Later in 1960, Coleman and Noll generalized the Rivlin-Ericksen model further to explain fluid with gradually fading memory [35]. This generalized version is widely known as Rivlin-Ericksen (RE) fluid and successfully explains flow of a wide class of fluids. The secondorder RE fluid comes with three independent transport coefficients: one first-order transport $n$ (shear viscosity) and two second-order transports, the first and second normal stress coefficients denoted by $\alpha_{1}$ and $\alpha_{2}$, respectively. For an equilibrium RE fluid (assuming specific Helmholtz free

\footnotetext{
${ }^{2}$ See Refs. [26-29] for null reduction of relativistic fluids.
}

TABLE I. Relation among transport coefficients of relativistic and nonrelativistic fluid.

\begin{tabular}{lc}
\hline \hline $\begin{array}{l}\text { Relativistic transport } \\
\text { coefficient }\end{array}$ & $\begin{array}{c}\text { Nonrelativistic transport } \\
\text { coefficient }\end{array}$ \\
\hline$\eta_{r}$ & $n=\eta_{r} u^{+}\left(1+\tau_{\pi} u^{+}(\partial . v)\right)$ \\
$\kappa=\frac{\eta_{r}}{T}$ \\
$\xi_{\sigma}$ & $\tilde{\xi}_{\sigma}=\frac{u^{+2}}{4}\left(\xi_{\sigma}-4 \eta_{r} \tau_{\pi}\right)$ \\
$\xi_{\omega}$ & $\tilde{\xi}_{\omega}=\frac{u^{+2}}{4} \xi_{\omega}$ \\
$\tau_{\omega}$ & $\tilde{\tau}_{\omega}=-\frac{u^{+2}}{2} \eta_{r}\left(\tau_{\pi}+\tau_{\omega}\right)$ \\
$\tau_{\pi}$ & $\tilde{\tau}_{\pi}=\eta_{r} u^{+2} \tau_{\pi}$ \\
\hline \hline
\end{tabular}

energy is minimum at equilibrium) satisfying the Claussius-Duhem inequality, Dunn and Fosdick [36] showed that both the shear viscosity, $n$ and the first normal coefficient $\alpha_{1}$ are positive, i.e., $n, \alpha_{1}>0$. In addition, the two second-order transports add up to zero, i.e., $\alpha_{1}+\alpha_{2}=0$. It has been further studied by Fosdick and Rajagopal [37] that shear viscosity and the first normal coefficient are always positive but the sum of the two second-order coefficients may not be zero always.

In this paper, we explore the relativistic origin of RivlinEricksen terms in the stress tensor of a non-Newtonian fluid. Starting with a generic second-order relativistic parity-odd uncharged fluid in $3+1$ dimensions, we reduce its constitutive relations over the light-cone and obtain a nonrelativistic parity-odd uncharged fluid in $2+1$ dimensions up to second order in derivative expansion. At first order, the reduced fluid has two independent transports: shear viscosity and thermal conductivity [18-20]. We observe that at second order there are in total eight independent terms in the stress tensor with four independent transports: $\tilde{\xi}_{\sigma}, \tilde{\xi}_{\omega}, \tilde{\tau}_{\omega}$, and $\tilde{\tau}_{\pi}$. Among these, the terms proportional to $\tilde{\xi}_{\sigma}$ and $\tilde{\tau}_{\pi}$ are present in the RE model. The RE fluid, therefore, is a subset of the generic second-order fluid that we have obtained in this paper. The relativistic source of the first RE term $\left(\tilde{\xi}_{\sigma}\right)$ is the square of the shear stress tensor. The origin of the second RE transport is interesting - it is proportional to the relaxation time of the relativistic fluid. Apart from the regular RE terms, we also obtain terms dependent on vorticity and temperature gradient at the second order.

We further compute the holographic values of the second-order transports using the fluid/gravity correspondence. We use the dictionary between relativistic and nonrelativistic transports (Table I) and use the holographic values of the relativistic ones to find the corresponding nonrelativistic values. We find that all four second-order transports are not independent. Three of them satisfy a universal relation, namely, $\tilde{\xi}_{\sigma}+\tilde{\tau}_{\omega}+\tilde{\tau}_{\pi}=0$, which is holographic and a generalization of the relation $\alpha 1+\alpha 2=0$, Ref. [36]. Furthermore, it is consistent with the observations of Ref. [37]. 
The plan of the paper is as follows. In Sec. II, we present the energy-momentum tensor of a relativistic second-order fluid. A short discussion about nonrelativistic fluid is given in Sec. III. In Sec. IV, we discuss light-cone reduction of relativistic fluid and explicitly compute the constitutive relations and transports of nonrelativistic fluid in terms of relativistic data up to second order in derivative expansion. We compare our nonrelativistic stress tensor with Rivlin-Ericksen fluid and find out the holographic values of nonrelativistic transports. Finally, we end our paper with concluding remarks and possible future directions in Sec. V.

\section{SECOND-ORDER RELATIVISTIC FLUID}

A relativistic fluid in $3+1$ dimensions without any chemical potential (uncharged fluid) is specified by six parameters: three velocity components (assuming $u^{\mu} u_{\mu}=$ $-1)$ and three local thermodynamic quantities [energy $E(x)$, temperature $T(x)$, and pressure $P(x)$ ]. However, local thermal equilibrium implies that the thermodynamic variables energy, temperature, and pressure satisfy the first law of thermodynamics at every spacetime point. Additionally, $E(x), P(x)$, and $T(x)$ also follow the equation of state and Euler relation $E+P=T S$, where $S$ is the local entropy density. As a result, a relativistic fluid is specified by three independent components of velocity and one thermodynamic variable, which we choose to be local temperature $T(x)$. The energy-momentum tensor of a relativistic fluid therefore depends on temperature and fluid velocities. To find an expression for the energymomentum tensor, we use its conservation equation

$$
\partial_{\mu} T^{\mu \nu}=0
$$

In $3+1$ dimensions, there is a total of four equations and we also have four unknown variables. Hence, the system is solvable. Since a fluid is considered to be in local thermal equilibrium and the length scale of variation of fluid variables is much, much larger than the mean free path of the system, hence, we express the energy-momentum tensor perturbatively in derivatives of fluid variables. At every order in derivative expansion, the energy-momentum tensor contains independent symmetric second-rank tensors constructed out of derivatives of fluid variables. If a fluid obeys any extra symmetry, we include that information in the energy-momentum tensor as well. For example, for a conformal fluid, the energy-momentum tensor is traceless. Therefore, while writing the independent terms at different orders, we take care of this constraint.

Relativistic hydrodynamics has been studied extensively in the last two decades in the context of the fluid/gravity correspondence and also independently. The energymomentum tensor of boundary gauge theory in flat spacetime has been constructed holographically by Refs. [38,39] up to second order in derivative expansion by solving Einstein's equation perturbatively. The secondorder corrected energy-momentum tensor and conserved current of a charged fluid are constructed in Refs. [1,2]. The authors in Ref. [8] give the most generic form of a second-order energy-momentum tensor and entropy current of a relativistic, conformal fluid in a Weyl covariant manner. The corresponding stress tensor contains all possible independent second-rank symmetric terms (up to second order) that are Weyl covariant, transverse, and traceless. The transport coefficients are also constrained due to the second law of thermodynamics. ${ }^{3}$

Following Ref. [8], the stress tensor of $(3+1)$ dimensional, uncharged, conformal fluid in a flat spacetime up to second order is given by

$$
\begin{aligned}
T^{\mu \nu}= & (E+P) u^{\mu} u^{\nu}+P \eta^{\mu \nu}-2 \eta_{r}\left(\sigma^{\mu \nu}-\tau_{\pi} \chi^{\mu \nu}-\tau_{\omega} T_{2 a}^{\mu \nu}\right) \\
& +\xi_{\sigma} T_{2 b}^{\mu \nu}+\xi_{\omega} T_{2 f}^{\mu \nu} .
\end{aligned}
$$

The first-order term $\sigma_{\mu \nu}$ is called the shear stress and is given by

$$
\sigma^{\mu \nu}=\frac{1}{2} P^{\mu \alpha} P^{\nu \beta}\left(\partial_{\alpha} u_{\beta}+\partial_{\beta} u_{\alpha}-\frac{2}{d+1} \partial . u\right) .
$$

The transport $\eta_{r}$ appearing at the first order is the shear viscosity coefficient. Since the fluid under consideration has scale invariance, the bulk viscosity coefficient is zero.

The second-order terms are given by

$T_{2 a}^{\mu \nu}=-\left(\omega_{\lambda}^{\mu}{ }_{\lambda} \sigma^{\lambda \nu}+\omega_{\lambda}^{\nu} \sigma^{\lambda \mu}\right)$,

$T_{2 b}^{\mu \nu}=\sigma_{\alpha}^{\mu} \sigma_{\alpha}^{\nu}-\frac{1}{3} P^{\mu \nu} \sigma^{\alpha \beta} \sigma_{\alpha \beta}$,

$T_{2 c}^{\mu \nu}=(\partial . u) \sigma^{\mu \nu}$,

$T_{2 d}^{\mu \nu}=\left(u . \partial u^{\mu}\right)\left(u . \partial u^{\nu}\right)-\frac{1}{3} P^{\mu \nu}\left(u . \partial u^{\alpha}\right)\left(u . \partial u_{\alpha}\right)$,

$T_{2 e}^{\mu \nu}=\frac{1}{2} P^{\mu \alpha} P^{\nu \beta} u^{\gamma} \partial_{\gamma}\left(\partial_{\alpha} u_{\beta}+\partial_{\beta} u_{\alpha}\right)-\frac{P^{\mu \nu}}{3} P^{\alpha \beta}(u . \partial)\left(\partial_{\alpha} u_{\beta}\right)$,

$T_{2 f}^{\mu \nu}=\omega_{\lambda}^{\mu} \omega^{\lambda \nu}+\frac{P^{\mu \nu}}{3} \omega^{\alpha \beta} \omega_{\alpha \beta}$,

$\chi^{\mu \nu}=\frac{1}{3} T_{2 c}^{\mu \nu}+T_{2 d}^{\mu \nu}+T_{2 e}^{\mu \nu}$.

The vorticity tensor $\omega^{\mu \nu}$ is

$$
\omega^{\mu \nu}=\frac{1}{2}\left(\partial^{\mu} u^{\nu}-\partial^{\nu} u^{\mu}+u^{\mu}(u . \partial) u^{\nu}-u^{\nu}(u . \partial) u^{\mu}\right),
$$

and the projector $P^{\mu \nu}$ is given by

$$
P^{\mu \nu}=\eta^{\mu \nu}+u^{\mu} u^{\nu} .
$$

\footnotetext{
${ }^{3}$ See Ref. [40] also.
} 
We start with this stress tensor and reduce it over the light cone to obtain the constitutive relations of a nonrelativistic fluid living in $2+1$ dimensions.

\section{NONRELATIVISTIC FLUID}

We now briefly review the nonrelativistic fluid living in flat $2+1$ dimensions. The dynamics of nonrelativistic uncharged fluid is governed by the following constitutive equations [41]:

(i) The continuity equation is

$$
\partial_{t} \rho+\partial_{i}\left(\rho v^{i}\right)=0
$$

where $\rho$ is the mass density and $v^{i}$ is the fluid velocity.

(ii) The conservation of momentum is

$$
\partial_{t}\left(\rho v^{i}\right)+\partial_{j} t^{i j}=0
$$

where $t^{i j}$ is the stress tensor.

(iii) The conservation of energy is

$$
\partial_{t}\left(\varepsilon+\frac{1}{2} \rho v^{2}\right)+\partial_{i} j^{i}=0,
$$

where $j^{i}$ is the energy current and $\varepsilon$ is the energy density.

These are in total four equations. There are six nonrelativistic fluid variables in $2+1$ dimensions: two components of velocity vector $v^{i}$, energy density $\varepsilon$, mass density $\rho$, temperature $\vartheta$, and pressure $p$. We again consider that the fluid is in local thermal equilibrium and thermodynamic variables obey the Euler relation and equation of state locally. Thus, we are left with four independent fluid variables. One may consider them to be fluid velocities, temperature, and pressure.

We shall later see that nonrelativistic fluid obtained under light-cone reduction is a restricted class of fluid [20,31]. Starting from the relativistic thermodynamic relations $d E=T d S$ (first law) and Euler relation $E+P=S T$, one obtains the usual nonrelativistic thermodynamics $d \varepsilon=$ $\vartheta d s+\rho_{m} d \rho$ (first law) and Euler equation $\varepsilon+p=s \vartheta+$ $\rho_{m} \rho$ if $\varepsilon+p+\rho_{m} \rho=0$. Here, $\rho_{m}$ is the mass chemical potential. The last equation puts a further restriction on the system. Using this equation, one can also consider the independent variables to be velocities, temperature, and mass chemical potential.

At ideal order, when there is no dissipation, the stress tensor and the energy current do not contain any derivatives and are solely expressed in terms of thermodynamic quantities and velocities. In the presence of dissipation, the stress tensor and energy current receive corrections proportional to the first derivative of velocities and temperature. The stress tensor and energy current of a compressible, uncharged, nonrelativistic fluid up to the first order in derivative expansion are given by [41]

$$
\begin{aligned}
t^{i j} & =\rho v^{i} v^{j}+p \eta^{i j}-n \tilde{\sigma}^{i j}, \\
j^{i} & =\left(\varepsilon+p+\frac{1}{2} \rho v^{2}\right) v^{i}+n v_{j} \tilde{\sigma}^{i j}-\kappa \partial^{i} \vartheta,
\end{aligned}
$$

where the dissipative stress tensor $\tilde{\sigma}_{i j}$ is given by

$$
\tilde{\sigma}^{i j}=\partial^{i} v^{j}+\partial^{j} v^{i}-\delta^{i j} \partial_{k} v^{k}
$$

$n$ is the shear viscosity coefficient, and $\kappa$ is the thermal conductivity. Since $\tilde{\sigma}_{i j}$ is proportional to derivative of velocity, the first-order fluid is called Newtonian fluid.

\section{A. Second grade non-Newtonian fluid}

There are several models for non-Newtonian fluids, both empirical and theoretical. The stress tensor of the simplest, second-order, incompressible, non-Newtonian fluid is given by Coleman and Noll [35],

$$
t_{i j}=-p \delta_{i j}+n \mathcal{A}_{i j}+\alpha_{1} \mathcal{B}_{i j}+\alpha_{2} \mathcal{A}^{i k} \mathcal{A}_{k}{ }^{j},
$$

where $\mathcal{A}$ and $\mathcal{B}$ are first and second Rivlin-Ericksen tensors [34] and are given by

$$
\begin{aligned}
& \mathcal{A}^{i j}=\partial^{i} v^{j}+\partial^{j} v^{i}, \\
& \mathcal{B}^{i j}=\partial^{i} a^{j}+\partial^{j} a^{i}+2 \partial^{i} v^{k} \partial^{j} v_{k},
\end{aligned}
$$

and

$$
a_{i}=\frac{\partial v^{i}}{\partial t}+v^{j} \partial_{j} v^{i}
$$

is the nonrelativistic acceleration. $n$ is the shear viscosity, and $\alpha_{1}$ and $\alpha_{2}$ are the first and second normal stress coefficients, respectively. It was shown in Ref. [36] that a RE fluid in thermal equilibrium satisfying Clausius-Duhem inequality has the following constraints on transports:

$$
n, \alpha_{1} \geq 0, \quad \text { and } \quad \alpha_{1}+\alpha_{2}=0 .
$$

The first condition ensures the positivity of local entropy production. Experimental results, however, show that there exist fluids which obey (12), but not the second relation in (15). It was further shown in Ref. [37] that stable fluids obey $\alpha_{1} \geq 0$ but the addition of $\alpha_{1}$ and $\alpha_{2}$ may not be zero always.

Another model of differential, second grade fluid is given by Huilgol [42]. In our notation, the corresponding stress tensor is

$$
\begin{aligned}
t_{i j}= & -p \delta_{i j}+n \mathcal{A}_{i j}+\alpha_{1}^{\prime}\left(\mathcal{B}_{i j}-\left(\tilde{\omega}^{i k} \mathcal{A}_{k}^{j}-\mathcal{A}^{i k} \tilde{\omega}_{k}^{j}\right)\right) \\
& +\alpha_{2} \mathcal{A}^{i k} \mathcal{A}_{k}^{j},
\end{aligned}
$$


where

$$
\tilde{\omega}^{i k}=\partial^{i} v^{k}-\partial^{k} v^{i}
$$

is the nonrelativistic vorticity tensor. In the subsequent section, we shall see that these two models of nonNewtonian fluid are subclass of a more generic secondorder nonrelativistic fluid obtained from its relativistic counterpart under light-cone reduction.

\section{LIGHT-CONE REDUCTION OF A SECOND-ORDER RELATIVISTIC FLUID}

Light-cone reduction of the relativistic conservation equation (1) works in the following way. We first write down the metric of $3+1$-dimensional flat spacetime as $d s^{2}=-2 d x^{+} d x^{-}+\sum_{i=1}^{2}\left(d x^{i}\right)^{2}$, where $x^{ \pm}$are light-cone directions. We then take the $x^{-}$direction to be an isometry direction; i.e., only those solutions of the relativistic energy-momentum tensor that do not depend on $x^{-}$are considered. Next, we identify the $x^{+}$direction with the nonrelativistic time. Expressing different components of (1) in light-cone coordinates and comparing them with (7), (8), and (9), we find the relations between nonrelativistic quantities and components of the relativistic energy-momentum tensor, valid at each order of derivative expansion $[18,25]$. The relations are given by

$$
\begin{aligned}
& T^{++}=\rho, \quad T^{+i}=\rho v^{i}, \quad T^{+-}=\varepsilon+\frac{1}{2} \rho v^{2}, \\
& T^{-i}=j^{i}, \quad T^{i j}=t^{i j} .
\end{aligned}
$$

Using these, different nonrelativistic constitutive relations were computed in Refs. [18-20] from relativistic fluid up to first order in derivative expansion. We shall follow a similar procedure to write down the second-order nonrelativistic constitutive relations.

\section{A. Second-order fluid}

Using the above reduction prescription, we compute nonrelativistic mass density, energy density, velocities, pressure, stress tensor, and energy current up to second order. Expressions are quite cumbersome. We present the expressions of the stress tensor in the main text. Other expressions (mass density, pressure, energy density, energy current, velocities, and mass chemical potential) have been provided in the Appendix.

The nonrelativistic stress ${ }^{4}$ tensor up to second order in derivative expansion is given by

\footnotetext{
${ }^{4}$ To derive the nonrelativistic stress tensor, we have used relativistic constraint equations (A1), and the identities $\frac{\eta_{r}}{u^{+2}}\left(\partial^{i} u^{+}-\frac{u^{+} \partial^{i} P}{E+P}\right)=-\kappa \partial^{i} \vartheta$ and $E+P=\vartheta s$.
}

$$
\begin{aligned}
t^{i j}= & p \delta^{i j}+\rho v^{i} v^{j}-n \tilde{\sigma}^{i j}+\tilde{\xi}_{\sigma} \tilde{\sigma}^{i k} \tilde{\sigma}_{k}^{j}+\tilde{\tau}_{\pi} \mathcal{B}^{i j} \\
& +\tilde{\tau}_{\omega}\left(\tilde{\omega}^{i k} \tilde{\sigma}_{k}^{j}-\tilde{\sigma}^{i k} \tilde{\omega}_{k}^{j}\right)+\tilde{\xi}_{\omega} \tilde{\omega}^{i k} \tilde{\omega}_{k}^{j} \\
& -\frac{\kappa^{2} \rho}{\vartheta_{s}}\left(\frac{1}{\vartheta_{s}}+\frac{1}{n^{2}}\left(\tilde{\xi}_{\omega}-\tilde{\xi}_{\sigma}-2\left(\tilde{\tau}_{\pi}+\tilde{\tau}_{\omega}\right)\right)\right) \partial^{i} \vartheta^{j} \vartheta \\
& +\frac{n \kappa}{\vartheta_{s}}\left(\partial^{i} \partial^{j} \vartheta+\partial^{j} \partial^{i \vartheta}\right)-4 \tilde{\xi}_{\omega} \frac{\rho}{\vartheta_{s}} a^{i} a^{j} \\
& +\frac{2 \kappa \rho}{\vartheta_{s}}\left(\frac{\tilde{\tau}_{w}}{n}-\frac{\tilde{\xi}_{\omega}}{n}+\frac{2 n}{\vartheta_{s}}\right)\left(a^{i} \partial^{j} \vartheta+a^{j} \partial^{j} \vartheta\right),
\end{aligned}
$$

where $a^{i}$ and $\tilde{\omega}^{i j}$ are the nonrelativistic acceleration and vorticity tensor given in by Eqs. (14) and (17), respectively. $\mathcal{B}^{i j}$ is second Rivlin-Ericksen tensor mentioned as before [Eq. (13)]. The expressions for $\rho, p$, and $v^{i}$ are given by (A3), (A7), and (A6), respectively. The nonrelativistic temperature $\vartheta$ is given by

$$
\vartheta=\frac{T}{u^{+}},
$$

and $s=\mathrm{Su}^{+}$is the nonrelativistic entropy density at the leading order. Nonrelativistic transports $n, \kappa, \tilde{\xi}_{\sigma}, \tilde{\xi}_{\omega}, \tilde{\tau}_{\pi}$, and $\tilde{\tau}_{\omega}$ are determined in terms of relativistic transports and other variables. The relations are given in Table I.

The first four second-derivative terms in the nonrelativistic stress tensor (19) are functions of derivatives of velocities. These four terms come under direct light-cone reduction of relativistic terms $\chi^{\mu \nu}, T_{2 a}^{\mu \nu}, T_{2 b}^{\mu \nu}$, and $T_{2 f}^{\mu \nu}$ terms in (2). Among these four terms, the first and the second terms are same as the second and the first Rivlin-Ericksen terms, ${ }^{5}$ respectively, with $\tilde{\xi}_{\sigma}=\alpha_{2}$ and $\tilde{\tau}_{\pi}=\alpha_{1}$. Thus, RE fluid is a subclass of (19). The Huilgol model (16) contains another term proportional to the product of vorticity and viscous stress tensor and is also present in (19) but comes with an independent transport $\tilde{\tau}_{\omega}$. Therefore, Huilgol fluid is another subclass of (19) with $\tilde{\tau}_{\omega}=-\tilde{\tau}_{\pi}=-\alpha_{1}^{\prime}$. It is mentioned in Sec. III that nonrelativistic fluid has two independent thermodynamic variables, and constitutive relations can depend on the derivatives of these two thermodynamic variables and velocities. Our light-cone reduced nonrelativistic fluid also contains these terms.

From Table I, we see that the second-order nonrelativistic transport coefficients satisfy the following relation:

$$
\tilde{\xi}_{\sigma}+\tilde{\tau}_{\omega}+\tilde{\tau}_{\pi}=\frac{u^{+2}}{4}\left(\xi_{\sigma}-2 \eta_{r}\left(\tau_{\pi}+\tau_{\omega}\right)\right)
$$

\section{Holographic nonrelativistic fluid}

Using the AdS/CFT correspondence Policastro et al. computed the holographic value of shear viscosity

\footnotetext{
${ }^{5}$ However, the light-cone reduced nonrelativistic fluid that we are considering is not incompressible.
} 
coefficient for a conformal fluid and showed that the ratio shear viscosity to entropy density for a holographic fluid is universal [43]. Holographic values of second-order transports were first computed by Refs. [38,39,44] independently. Universality of second-order relativistic transports of conformal, nonconformal holographic fluid, and fluid with higher-derivative gravity dual are discussed in Refs. [45-50].

The holographic values of nonrelativistic transports can be computed in two different ways. One can use the dictionary between the nonrelativistic and relativistic transports (Table I) and then use the holographic values of relativistic transports computed in Refs. $[38,39,44]$. In the second approach, one constructs the holographic dual of a Schrödinger fluid using the TsT transformations and compute the transports by writing down the boundary stress tensor. These two methods are equivalent up to first order in derivative expansion [31]. The first-order nonrelativistic transports were computed in Refs. [18,19] using the first method. Here, we follow the same method to compute the holographic values of second-order transports.

It is shown in Refs. [45,46] that in any arbitrary dimensions $d>2$ uncharged relativistic conformal fluids that admit a gravity dual respect a universal relation among the second-order transport coefficients,

$$
\xi_{\sigma}=2 \eta_{r}\left(\tau_{\omega}+\tau_{\pi}\right)
$$

This relation can easily be checked by computing the transports in anti-de Sitter (AdS)-Schwarzschild background $[38,39,45]$,

$$
\begin{aligned}
\xi_{\sigma} & =\frac{4 \eta_{r}}{2 \pi T}, \quad \tau_{\pi}=\frac{2-\log 2}{2 \pi T}, \\
\tau_{\omega} & =\frac{\log 2}{2 \pi T} \quad \text { and } \quad \xi_{\omega}=0 .
\end{aligned}
$$

The holographic constraint on relativistic transports poses restriction on nonrelativistic transport that stemmed from a holographic relativistic fluid. Using (21), we find that the second-order nonrelativistic transports satisfy

$$
\tilde{\xi}_{\sigma}+\tilde{\tau}_{\omega}+\tilde{\tau}_{\pi}=0
$$

On this constraint, the stress tensor (19) is given by

$$
\begin{aligned}
t^{i j}= & \rho v^{i} v^{j}-n \tilde{\sigma}^{i j}+p \delta^{i j}+\tilde{\xi}_{\sigma} \tilde{\sigma}^{i k} \tilde{\sigma}_{k}^{j}+\tilde{\tau}_{\pi} \mathcal{B}^{i j} \\
& +\tilde{\tau}_{\omega}\left(\tilde{\omega}^{i k} \tilde{\sigma}_{k}^{j}-\tilde{\sigma}^{i k} \tilde{\omega}_{k}^{j}\right)+\frac{2 n \kappa}{\vartheta_{s}} \partial^{i} \partial^{j} \vartheta \\
& -\frac{\kappa^{2} \rho}{\vartheta_{s}}\left(\frac{1}{\vartheta_{s}}+\frac{\tilde{\xi}_{\sigma}}{n^{2}}\right) \partial^{i} \vartheta \partial^{j} \vartheta \\
& +\frac{2 \kappa \rho}{\vartheta_{s}}\left(\frac{\tilde{\tau}_{w}}{n}+\frac{2 n}{\vartheta_{s}}\right)\left(a^{i} \partial^{j} \vartheta+a^{j} \partial^{j} \vartheta\right) .
\end{aligned}
$$

Comparing (25) with the stress tensor of RE fluid (12), we find

$\alpha_{1}=\tilde{\tau}_{\pi}=\frac{n}{2 \pi \vartheta}(2-\log 2), \quad \alpha_{2}=\tilde{\xi}_{\sigma}=\frac{n}{2 \pi \vartheta}(\log 2-1)$.

Holographic values of other second-order nonrelativistic transports are given by

$$
\tilde{\tau}_{\omega}=-\frac{n}{2 \pi \vartheta} \quad \text { and } \quad \tilde{\xi}_{\omega}=0
$$

The stress tensor (25) is a generalized version of the Huilgol stress tensor (16) with $\tilde{\tau}_{\omega}=-\tilde{\tau}_{\pi}$. The constraint relation (24) is the generalization of the relation given by Dunn and Fosdick (15). Apart from the regular velocity dependent terms, our expression (25) also contains terms dependent on derivatives of temperature. Thus, we have constructed the most generic holographic nonrelativistic stress tensor corrected up to second order in the derivative expansion.

As mentioned in Sec. III, the light-cone reduced nonrelativistic fluid belongs to a restricted class where the mass chemical potential $\rho_{m}$ follows the relation $\varepsilon+p+\rho_{m} \rho=0$. One can use this relation to find the mass chemical potential corrected up to second order (A10). $\rho_{m}$ does not receive any correction at first order as before [20]; however, it has corrections at the second order.

The AdS/CFT correspondence relates a strongly coupled gauge theory to a weakly coupled gravity in AdS space. Therefore, the holographic relation (24) puts a constraint on the nonrelativistic fluid in the strong coupling regime. Although the relation (24) is an interesting generalization of Dunn and Fosdick (15), the physical origin of such a relation is not clear. Even the relativistic counterpart (22) appears to be a mathematical outcome of holographic construction. There is no first principle or symmetry argument behind such a relation. Therefore, it would be interesting to understand the physical origin of this constraint relation. Since a dissipative fluid system produces entropy locally, the divergence of the entropy current is positive. It would be interesting if one could reproduce such a constraint on the second-order transports from the positivity of the local nonrelativistic entropy current.

\section{CONCLUSION}

In this paper, we have studied the holographic constraint on second-order transports of an uncharged nonrelativistic fluid obtained from a relativistic uncharged fluid with Weyl invariance by light-cone reduction. The resultant holographic stress tensor has seven terms at the second order with four transports. We also compute the holographic values of these second-order transports and observe that 
three of them satisfy a universal relation: the sum of the three coefficients is zero. The expression of the nonrelativistic stress tensor obtained from its relativistic counterpart is a generalization of two well-known Rheological models, namely, the Rivlin-Ericksen and Huilgol models. Since the AdS/CFT dictionary relates a strongly coupled gauge theory to a weakly coupled gravity in AdS space, the holographic constraint (24) is valid in the strong coupling regime. In this paper, we have explicitly reduced a $3+1-$ dimensional relativistic fluid to a $2+1$-dimensional nonrelativistic fluid. However, our construction holds for relativistic fluid in any arbitrary dimensions. We picked up 3+1-dimensional relativistic fluid as our starting point because we also intend to reduce the corresponding holographic setup in $4+1$ dimensions following Ref. [31].

Light-cone reduction of nonconformal charged fluid up to first order in derivative expansion is considered in Ref. [20]. Relativistic nonconformal, uncharged fluid has been studied up to third order in derivative expansion [40,51,52], whereas the relativistic charged fluid is studied up to second order [53]. One can start with a relativistic nonconformal fluid of higher order. Such a fluid will come with more transports in the first and second orders. The light-cone reduction principle will be the same for such a fluid. However, the reduced nonrelativistic fluid will also have extra transports. In this paper, we have focused on the conformal sector because the holographic constraint (22) relates the transports in the conformal sector. Considering the reduction of the nonconformal fluid is an interesting generalization of this work, and we leave it as a future open problem.

As mentioned in the Introduction, there are several ways to deduce a nonrelativistic system from a relativistic one. The most common technique is to consider the speed of light $c$ to be infinity. In this limit, the Lorentz transformations boil down to the Galilean transformations. On the other hand, the light-cone reduction reduces the Lorentz algebra directly to Galilean algebra. These two reduction prescriptions have completely different mechanisms when applied to fluid dynamics. A priory there is no reason to expect that the nonrelativistic constitutive relations obtained by applying these two techniques will match. It is shown by Ref. [16] that the light-cone reduction (LCR) and large $c$ expansion render the same nonrelativistic constitutive relations in the parity-even sector. However, they differ in the parity-odd sector [14]. In either case, the authors considered constitutive relations only up to the first order in derivative. It would definitely be interesting to obtain second-order nonrelativistic constitutive relations using the large $c$ expansion technique and compare that with the result we obtained in this paper. This, however, goes beyond the scope of our paper, and we thus keep it for a future endeavor.

Our results open up the platform to study the secondorder stress tensor for a nonrelativistic charged fluid under light-cone reduction of charged, second-order relativistic fluid. The construction of second-order constitutive relations of the nonrelativistic fluids from a nonrelativistic gravity dual is another important problem to look at. Second-order entropy current for uncharged/charged nonrelativistic fluid can also be studied. It would be interesting to find the constraints on second-order nonrelativistic transports imposed by the second law of thermodynamics.

\section{ACKNOWLEDGMENTS}

We would like to thank Nabamita Banerjee, Sayantani Bhattacharyya, and Debangshu Mukherjee for insightful discussions. The work of S. D. is supported by Grants No. EMR/2016/006294 and No. MTR/2019/000390 from the SERB, Government of India. S. D. also acknowledges the Simons Associateship of the Abdus Salam ICTP, Trieste, Italy.

\section{APPENDIX: DETAILED CALCULATION OF NONRELATIVISTIC QUANTITIES}

The first-order relativistic fluid satisfies the following relations:

$$
\begin{aligned}
(u . \partial) E+(E+P)(\partial . u) & =\eta_{r}\left(\partial_{\mu} u_{\nu}+\partial_{\nu} u_{\mu}\right) \sigma^{\mu \nu}, \\
P^{\mu \nu} \partial_{\mu} P+(E+P)(u . \partial) u^{\nu} & =2 \eta_{r}\left(\partial_{\mu} \sigma^{\mu \nu}-u^{\nu} \sigma_{\alpha \beta} \sigma^{\alpha \beta}\right) .
\end{aligned}
$$

The relativistic fluid velocity is normalized: $u^{\mu} u_{\mu}=-1$. We use this relation to replace the $u^{-}$component in terms of other components,

$$
u^{-}=\frac{1}{2 u^{+}}\left(1+u_{k} u^{k}\right)
$$

Computing the ++ component of the relativistic energymomentum tensor (2) and identifying that with the nonrelativistic mass density $\rho=T^{++}$, we obtain

$$
\begin{aligned}
\rho= & (E+P)\left(u^{+}\right)^{2}-\frac{2 \eta_{r}^{2} u^{+}}{E+P} \partial_{k} \mathcal{Y}^{k} \\
& -\frac{u^{+2}}{3}\left(\xi_{\sigma}-\frac{16 \eta_{r}^{2}}{E+P}\right) \sigma_{\alpha \beta} \sigma^{\alpha \beta}+\frac{\xi_{\omega}}{3} u^{+2} \omega_{\alpha \beta} \omega^{\alpha \beta} \\
& +\left(2 \eta_{r}\left(\tau_{\pi}+\tau_{\omega}\right)-\xi_{\omega}\right) \frac{u^{+} \mathcal{Y}_{k} \partial^{k} P}{E+P} \\
& +\left(4 \eta_{r} \tau_{\omega}+\xi_{\sigma}-\xi_{\omega}\right) \frac{\mathcal{Y}_{k} \mathcal{Y}^{k}}{4}-\xi_{\omega} \frac{u^{+2} \partial_{k} P \partial^{k} P}{(E+P)^{2}}
\end{aligned}
$$

where

$$
\mathcal{Y}^{\alpha}=\mathcal{Y}^{\alpha+}=\partial^{\alpha} u^{+}+u^{+}(u . \partial) u^{\alpha}-\frac{u^{+} u^{\alpha}}{3}(\partial . u),
$$

and 


$$
\mathcal{Y}^{\mu \nu}=\partial^{\mu} u^{\nu}+u^{\nu} u^{\gamma} \partial_{\gamma} u^{\mu}-\frac{1}{3} u^{\mu} u^{\nu}(\partial . u)
$$

At first order, it reduces to $\mathcal{Y}^{\alpha}=\left(\partial^{\alpha} u^{+}-\frac{u^{+} \partial^{\alpha} P}{E+P}\right)$. As before, the nonrelativistic mass density does not receive any correction at first order for the conformal fluid $[1,18]$.

We now use the mapping $T^{+i}=\rho v^{i}$ to compute the nonrelativistic velocity in terms of relativistic data. Computing the $+i$ component of $T^{\mu \nu}$, we find

$$
\begin{aligned}
v^{i}= & \frac{u^{i}}{u^{+}}-\frac{\eta_{r}}{\rho} \mathcal{Y}^{i}+\frac{\eta_{r}^{2} u^{+2}}{\rho^{2}}\left(\partial_{k} \mathcal{Y}^{k}-\frac{8}{3} u^{+} \sigma_{\alpha \beta} \sigma^{\alpha \beta}\right) u^{i}+\frac{\eta_{r}}{\rho} \tau_{\omega}\left(\left(\mathcal{Y}^{k}+\frac{u^{+} \partial^{k} P}{E+P}\right)\left(\partial_{k} u^{i}-\frac{u^{i}}{u^{+}} \mathcal{Y}_{k}-\frac{u^{i} \partial_{k} P}{E+P}\right)\right. \\
& \left.+\left(u . \partial P-u^{k} \partial_{k} P\right) \frac{\mathcal{Y}^{i}}{E+P}+\frac{u^{+}}{E+P}\left(2 u . \partial P-u^{k} \partial_{k} P\right) \frac{\partial^{i} P}{E+P}+u^{+} \frac{\partial^{i} u^{k} \partial_{k} P}{E+P}\right) \\
& +\frac{\xi_{\sigma}}{4 \rho}\left(\mathcal{Y}_{+} \mathcal{Y}^{i}+\frac{4 u . \partial P}{E+P} \mathcal{Y}^{i}+\mathcal{Y}_{k}\left(\partial^{k} u^{i}+\partial^{i} u^{k}-\frac{u^{k} \partial^{i} P}{E+P}\right)-\mathcal{Y}_{k}\left(\mathcal{Y}^{k}+\frac{u^{+} \partial^{k} P}{E+P}\right) \frac{u^{i}}{u^{+}}\right) \\
& +\frac{\xi_{\omega}}{4 \rho}\left(\left(\mathcal{Y}_{+}+2 \frac{u^{+} \partial_{+} P}{E+P}\right) \mathcal{Y}^{i}-\left(\mathcal{Y}^{k}+2 \frac{u^{+} \partial^{k} P}{E+P}\right)\left(\partial_{k} u^{i}-\partial^{i} u_{k}\right)\right. \\
& \left.+\frac{\partial^{i} P}{E+P}\left(u_{k} \mathcal{Y}^{k}+2 u^{+} \mathcal{Y}_{+}+\frac{2 u^{+}}{E+P}\left(2 u . \partial P-u^{k} \partial_{k} P\right)\right)+\left(\mathcal{Y}^{k} \mathcal{Y}_{k}+3 u^{+} \frac{\mathcal{Y}^{k} \partial_{k} P}{E+P}+2 u^{+2} \frac{\partial_{k} P \partial^{k} P}{(E+P)^{2}}\right) \frac{u^{i}}{u^{+}}\right) \\
& +\frac{\eta_{r}}{\rho} \tau_{\pi}\left(u . \partial \mathcal{Y}^{i}+\frac{u^{+} \partial^{k} P}{E+P}\left(\partial^{k} u^{i}-\frac{u^{i} \partial^{k} P}{E+P}\right)-\frac{u . \partial P}{E+P}\left(\mathcal{Y}^{i}-\frac{u^{+} \partial^{i} P}{E+P}\right)-u^{i} \frac{\mathcal{Y}_{k} \partial^{k} P}{E+P}+u^{+} \frac{\partial^{\alpha} P \partial^{i} u_{\alpha}}{E+P}\right) .
\end{aligned}
$$

Second-order corrected nonrelativistic equilibrium pressure in terms of relativistic variables can be obtained from the diagonal terms in $T^{i j}$ and is given by

$$
\begin{aligned}
p= & P+\frac{\eta_{r}^{2}}{\rho}\left(2 u^{+} \partial_{k} \mathcal{Y}^{k}-\frac{8}{3} u^{+2} \sigma_{\alpha \beta} \sigma^{\alpha \beta}-2 \mathcal{Y}_{k} \mathcal{Y}^{k}+6 u^{+3} a_{k} \mathcal{Y}^{k}\right) \\
& -\eta_{r} \tau_{\pi} u^{+2}\left(\partial . a-\partial_{k} v^{m} \partial_{m} v^{k}+(\partial . v)^{2}\right)-\frac{\xi_{\sigma}}{3} \sigma_{\alpha \beta} \sigma^{\alpha \beta}+\frac{\xi_{\omega}}{3} \omega_{\alpha \beta} \omega^{\alpha \beta} .
\end{aligned}
$$

Computation of the +- component of the relativistic stress tensor and the identification $T^{+-}=\varepsilon+\frac{1}{2} \rho v^{2}$ give us the nonrelativistic energy density

$$
\begin{aligned}
\varepsilon= & \frac{E-P}{2}-\frac{1}{2 u^{+2}}\left(\frac{\eta_{r}{ }^{2}}{(E+P)}+\eta_{r} \tau_{\omega}+\frac{\xi_{\sigma}-\xi_{\omega}}{4}\right) \mathcal{Y}_{k} \mathcal{Y}^{k}-\frac{1}{2 u^{+2}}\left[-2 \frac{\eta_{r}^{2} u^{+}}{E+P} \partial_{k} \mathcal{Y}^{k}+\left(2 \eta_{r}\left(\tau_{\pi}+\tau_{\omega}\right)-\xi_{\omega}\right) u^{+} \frac{\mathcal{Y}_{k} \partial^{k} P}{E+P}\right. \\
& \left.-\frac{u^{+2}}{3}\left(\xi_{\sigma}-\frac{16 \eta_{r}^{2}}{E+P}\right) \sigma_{\alpha \beta} \sigma^{\alpha \beta}+\frac{\xi_{\omega}}{3} u^{+2} \omega_{\alpha \beta} \omega^{\alpha \beta}-\xi_{\omega} u^{+2} \frac{\partial_{k} P \partial^{k} P}{(E+P)^{2}}\right] .
\end{aligned}
$$

The nonrelativistic energy current density can be computed by identifying $T^{-i}=j^{i}$. It is given by

$$
\begin{aligned}
j^{i}= & \left(\varepsilon+p+\frac{1}{2} \rho v^{2}\right) v^{i}-n v_{k} \tilde{\sigma}^{k i}-\kappa\left(1+\frac{3 n}{\vartheta_{s}}(\partial . v)\right) \partial^{i} \vartheta+\frac{\kappa}{n}\left(\tilde{\xi}_{\sigma}-\tilde{\tau}_{\omega}\right) \partial_{k} \vartheta \tilde{\sigma}^{k i}-\left(\frac{n^{2}}{\vartheta_{s}}+2 \tilde{\tau}_{\omega}\right) a_{k} \tilde{\sigma}^{k i} \\
& +\frac{n \kappa}{\vartheta_{s}} v_{k}\left(\partial^{k} \partial^{i} \vartheta+\partial^{i} \partial^{k} \vartheta\right)-\frac{\kappa}{n}\left(\frac{n^{2}}{\vartheta_{s}}+\tilde{\xi}_{\omega}+\tilde{\tau}_{\omega}\right) \partial_{k} \vartheta \omega^{k i}+\tilde{\xi}_{\omega} v_{k} \tilde{\omega}^{k l} \tilde{\omega}_{l}^{i}-\frac{\kappa^{2} \rho}{\vartheta_{s}}\left(\frac{1}{\vartheta_{s}}+\frac{\tilde{\xi}_{\omega}-\tilde{\xi}_{\sigma}-2\left(\tilde{\tau}_{\pi}+\tilde{\tau}_{\omega}\right)}{n^{2}}\right) v_{k} \partial^{k} \vartheta \partial^{i} \vartheta \\
& -4 \tilde{\xi}_{\omega} \frac{\rho}{\vartheta_{s}} a_{k} v^{k} a^{i}+\tilde{\tau}_{\pi} v_{k} \mathcal{B}^{k i}+\tilde{\xi}_{\sigma} v_{k} \tilde{\sigma}^{k l} \tilde{\sigma}_{l}^{i}+\frac{2 \kappa \rho}{n \vartheta s}\left(\frac{2 n^{2}}{\vartheta_{s}}+\tilde{\tau}_{\omega}-\tilde{\xi}_{\omega}\right) v_{k}\left(\partial^{k} \vartheta a^{i}+\partial^{i} \vartheta a^{k}\right)-2 \tilde{\xi}_{\omega} a_{k} \tilde{\omega}^{k i} \\
& +\tilde{\tau}_{\omega} v_{k}\left(\omega^{k l} \tilde{\sigma}_{l}^{i}-\tilde{\sigma}^{k l} \omega_{l}^{i}\right)-\left(\tilde{\tau}_{\pi}-\frac{n^{2}}{\vartheta_{s}}\right)\left(\frac{\vartheta s}{\rho}\right) \partial^{i}(\partial . v)+\frac{n^{2}}{\rho} \partial_{k} \tilde{\sigma}^{k i} .
\end{aligned}
$$


Using the relation $\varepsilon+p+\rho_{m} \rho=0$, we find the mass chemical potential as follows:

$$
\begin{aligned}
\rho_{m}= & -\frac{1}{2 u^{+2}}+\frac{2 n^{2}}{\vartheta^{2} s^{2} u^{+2}} \tilde{\sigma}^{k l} \tilde{\sigma}_{k l}+\frac{\tilde{\tau}_{\pi}}{\vartheta s u^{+2}}\left((\partial . v)^{2}-\partial_{k} v^{m} \partial_{m} v^{k}\right)+\left(\frac{4 n^{2}}{\vartheta_{s}}-\tilde{\tau}_{\pi}\right) \frac{\partial_{k} \partial^{k} \vartheta}{\vartheta^{2} s u^{+4}} \\
& +\left(\tilde{\tau}_{\pi}+2 \tilde{\tau}_{\omega}-\tilde{\xi}_{\omega}+\tilde{\xi}_{\sigma}-\frac{7 n^{2}}{2 \vartheta s}\right) \frac{\partial_{k} \vartheta \partial^{k} \vartheta}{\vartheta^{3} s u^{+4}}+\left(2 \tilde{\tau}_{\pi}+4 \tilde{\tau}_{\omega}-4 \tilde{\xi}_{\omega}-\frac{2 n^{2}}{\vartheta_{s}}\right) \frac{\partial_{k} \vartheta \partial^{k} u^{+}}{\vartheta^{2} s u^{+3}} \\
& -\frac{\tilde{\tau}_{\pi}}{\vartheta_{s} u^{+5}} \partial_{k} \partial^{k} u^{+}+\frac{3 \tilde{\tau}_{\pi}-4 \tilde{\xi}_{\omega}}{\vartheta s u^{+6}} \partial_{k} u^{+} \partial^{k} u^{+} .
\end{aligned}
$$

[1] N. Banerjee, J. Bhattacharya, S. Bhattacharyya, S. Dutta, R. Loganayagam, and P. Surowka, Hydrodynamics from charged black branes, J. High Energy Phys. 01 (2011) 094.

[2] J. Erdmenger, M. Haack, M. Kaminski, and A. Yarom, Fluid dynamics of R-charged black holes, J. High Energy Phys. 01 (2009) 055.

[3] D. T. Son and P. Surowka, Hydrodynamics with Triangle Anomalies, Phys. Rev. Lett. 103, 191601 (2009).

[4] W. Israel, Nonstationary irreversible thermodynamics: A causal relativistic theory, Ann. Phys. (N.Y.) 100, 310 (1976).

[5] W. Israel and J. M. Stewart, Transient relativistic thermodynamics and kinetic theory, Ann. Phys. (N.Y.) 118, 341 (1979).

[6] W. A. Hiscock and L. Lindblom, Stability and causality in dissipative relativistic fluids, Ann. Phys. (N.Y.) 151, 466 (1983).

[7] P. Kovtun, First-order relativistic hydrodynamics is stable, J. High Energy Phys. 10 (2019) 034.

[8] R. Loganayagam, Entropy current in conformal hydrodynamics, J. High Energy Phys. 05 (2008) 087.

[9] B. Julia and H. Nicolai, Null Killing vector dimensional reduction and Galilean geometrodynamics, Nucl. Phys. B439, 291 (1995).

[10] M. Hassaine and P. A. Horvathy, Field dependent symmetries of a nonrelativistic fluid model, Ann. Phys. (N.Y.) 282, 218 (2000).

[11] K. Jensen and A. Karch, Revisiting non-relativistic limits, J. High Energy Phys. 04 (2015) 155.

[12] M. Geracie, K. Prabhu, and M. M. Roberts, Fields and fluids on curved non-relativistic spacetimes, J. High Energy Phys. 08 (2015) 042.

[13] K. Jensen, On the coupling of Galilean-invariant field theories to curved spacetime, SciPost Phys. 5, 011 (2018).

[14] K. Jensen, Aspects of hot Galilean field theory, J. High Energy Phys. 04 (2015) 123.

[15] A. Lucas and P. Surówka, Phenomenology of nonrelativistic parity-violating hydrodynamics in $2+1$ dimensions, Phys. Rev. E 90, 063005 (2014).

[16] M. Kaminski and S. Moroz, Nonrelativistic parity-violating hydrodynamics in two spatial dimensions, Phys. Rev. B 89, 115418 (2014).

[17] A. Jain, Galilean anomalies and their effect on hydrodynamics, Phys. Rev. D 93, 065007 (2016).
[18] M. Rangamani, S. F. Ross, D. T. Son, and E. G. Thompson, Conformal non-relativistic hydrodynamics from gravity, J. High Energy Phys. 01 (2009) 075.

[19] D. K. Brattan, Charged, conformal non-relativistic hydrodynamics, J. High Energy Phys. 10 (2010) 015.

[20] N. Banerjee, S. Dutta, A. Jain, and D. Roychowdhury, Entropy current for non-relativistic fluid, J. High Energy Phys. 08 (2014) 037.

[21] C. R. Hagen, Scale and conformal transformations in galilean-covariant field theory, Phys. Rev. D 5, 377 (1972).

[22] C. Duval, G. Burdet, H. P. Kunzle, and M. Perrin, Bargmann structures and Newton-Cartan theory, Phys. Rev. D 31, 1841 (1985).

[23] C. Duval, M. Hassaine, and P. A. Horvathy, The geometry of Schrodinger symmetry in gravity background/non-relativistic CFT, Ann. Phys. (Amsterdam) 324, 1158 (2009).

[24] C. Duval and P. A. Horvathy, Non-relativistic conformal symmetries and Newton-Cartan structures, J. Phys. A 42, 465206 (2009).

[25] M. Rangamani, Holography for non-relativistic CFTs, Acta Phys. Pol. B 40, 3745 (2009), https://www.actaphys.uj.edu .pl/R/40/12/3745/pdf.

[26] N. Banerjee, S. Dutta, and A. Jain, Null fluids-A new viewpoint of galilean fluids, Phys. Rev. D 93, 105020 (2016).

[27] N. Banerjee, S. Dutta, and A. Jain, Equilibrium partition function for nonrelativistic fluids, Phys. Rev. D 92, 081701 (2015).

[28] N. Banerjee, S. Dutta, and A. Jain, First order galilean superfluid dynamics, Phys. Rev. D 96, 065004 (2017).

[29] N. Banerjee, S. Atul Bhatkar, and A. Jain, Second order Galilean fluids and Stokes' law, Phys. Rev. D 97, 096018 (2018).

[30] S. F. Ross and O. Saremi, Holographic stress tensor for nonrelativistic theories, J. High Energy Phys. 09 (2009) 009.

[31] S. Dutta and H. Krishna, Light-cone reduction vs. TsT transformations: A fluid dynamics perspective, J. High Energy Phys. 05 (2018) 029.

[32] M. Reiner, A mathematical theory of dilatancy, Am. J. Math. 67, 350 (1945).

[33] R. S. Rivlin, The hydrodynamics of non-Newtonian fluids, Proc. R. Soc. A 193, 260 (1948).

[34] R. S. Rivlin and J. L. Ericksen, Stress deformation relations for isotropic materials, J. Ration. Mech. Anal. 4, 323 (1955). 
[35] B. D. Coleman and W. Noll, An approximation theorem for functionals with applications in continuum mechanics, Arch. Ration. Mech. Anal. 6, 355 (1960).

[36] J. E. Dunn and R. L. Fosdick, Thermodynamics, stability and boundedness of fluids of complexity 2 and fluids of second grade, Arch. Ration. Mech. Anal. 56, 191 (1974).

[37] R. L. Fosdick and K. R. Rajagopal, Anomalous features in the model of second order fluids, Arch. Ration. Mech. Anal. 70, 145 (1979).

[38] S. Bhattacharyya, V. E. Hubeny, S. Minwalla, and M. Rangamani, Nonlinear fluid dynamics from gravity, J. High Energy Phys. 02 (2008) 045.

[39] R. Baier, P. Romatschke, D. T. Son, A. O. Starinets, and M. A. Stephanov, Relativistic viscous hydrodynamics, conformal invariance, and holography, J. High Energy Phys. 04 (2008) 100.

[40] S. Bhattacharyya, Constraints on the second order transport coefficients of an uncharged fluid, J. High Energy Phys. 07 (2012) 104.

[41] L. D. Landau and E. M. Lifshitz, Fluid Mechanics (Pergamon Press, New York, 1997).

[42] R. R. Huilgol, A second order fluid of differential type, Int. J. Nonlinear Mech. 3, 471 (1968).

[43] G. Policastro, D. T. Son, and A. O. Starinets, The Shear Viscosity of Strongly Coupled $N=4$ Supersymmetric Yang-Mills Plasma, Phys. Rev. Lett. 87, 081601 (2001).

[44] M. Natsuume and T. Okamura, Causal hydrodynamics of gauge theory plasmas from AdS/CFT duality, Phys. Rev. D 77, 066014 (2008).
[45] S. Bhattacharyya, R. Loganayagam, I. Mandal, S. Minwalla, and A. Sharma, Conformal nonlinear fluid dynamics from gravity in arbitrary dimensions, J. High Energy Phys. 12 (2008) 116.

[46] M. Haack and A. Yarom, Universality of second order transport coefficients from the gauge-string duality, Nucl. Phys. B813, 140 (2009).

[47] E. Shaverin and A. Yarom, Universality of second order transport in Gauss-Bonnet gravity, J. High Energy Phys. 04 (2013) 013.

[48] S. Grozdanov and A. O. Starinets, On the universal identity in second order hydrodynamics, J. High Energy Phys. 03 (2015) 007.

[49] P. Kleinert and J. Probst, Second-order hydrodynamics and universality in non-conformal holographic fluids, J. High Energy Phys. 12 (2016) 091.

[50] S. Grozdanov and A. O. Starinets, Second-order transport, quasinormal modes and zero-viscosity limit in the GaussBonnet holographic fluid, J. High Energy Phys. 03 (2017) 166.

[51] P. Romatschke, Relativistic viscous fluid dynamics and nonequilibrium entropy, Classical Quantum Gravity 27, 025006 (2010).

[52] S. Grozdanov and N. Kaplis, Constructing higher-order hydrodynamics: The third order, Phys. Rev. D 93, 066012 (2016).

[53] S. Lahiri, Second order causal hydrodynamics in Eckart frame: Using gradient expansion scheme, Classical Quantum Gravity 37, 075010 (2020). 